\title{
Extraction and Determination of Nicotine in Tobacco from Selected Local Cigarettes Brands in Iraq
}

\author{
Wedad H. Al-Dahhan ${ }^{1}$, Mohammed Kadhom 2 ${ }^{(\mathbb{D})}$, Emad Yousif ${ }^{1, *}{ }^{\mathbb{C}}$, Salam A. Mohammed ${ }^{3(\mathbb{D})}$, Ayad \\ Alkaim 4 iD \\ 1 Department of Chemistry, College of Science, Al-Nahrain University, Baghdad, Iraq \\ 2 Department of Environmental Science, College of Energy and Environmental Sciences, Alkarkh University of Science, \\ Baghdad, Iraq \\ 3 Department of Chemical and Petrochemical Engineering, College of Engineering and Architecture, University of Nizwa, \\ 616 Nizwa, Sultanate of Oman \\ 4 Department of Chemistry Science, Faculty of Science for Women (SCIW), University of Babylon, Babylon, Iraq \\ * Correspondence: emad_yousif@ hotmail.com;
}

Received: 11.01.2021; Revised: 10.05.2021; Accepted: 15.05.2021; Published: 27.06.2021

\begin{abstract}
Tobacco smoke contains more than 3,800 different compounds, where all could harm the exposed humans to different degrees. Among them, nicotine (3-(1-methyl-2-pyrrolidinyl) pyridine) is considered the most toxic compound. In many countries, tobacco smoking is considered a severe health hazard and a significant factor in death and several common diseases. Because of that, knowing the toxicity of nicotine is important to help understand tobacco-induced human diseases and identify the potential risks associated with the therapeutic use of nicotine as an aid in smoking cessation. Thereby, estimating the nicotine amount is very crucial in tobacco production. As this work aims to extract and determine nicotine, which is the highest toxic component in tobacco plant leaves, several methods were carried out to extract the nicotine. All extracting methods were based on water extraction as a polar solvent and the other non-polar solvents. Water shows a limited extraction activity for nicotine from tobacco leaves. Since nicotine is a dibasic compound, an alkaline solution $(40 \% \mathrm{NaOH})$ was used to extract nicotine from tobacco leaves for the selected samples. Further, solvent extraction by non-polar solvents was conducted to extract nicotine from the alkaline solution and prepare it for the spectrometric analysis. FT-IR and UV analysis show that the oily substance, which was extracted from tobacco leaves, is nicotine. Results were confirmed by microscopic examination for the extracted nicotine after the addition of mercuric chloride, where flowery shape crystals of nicotine liganded with mercuric chloride complex were formed.
\end{abstract}

Keywords: nicotine; tobacco; solvent extraction; cigarette; smoking.

(C) 2021 by the authors. This article is an open-access article distributed under the terms and conditions of the Creative Commons Attribution (CC BY) license (https://creativecommons.org/licenses/by/4.0/).

\section{Introduction}

Tobacco smoke is a combination of around 5,000 chemicals with dynamic and reactive properties [1]. These components are generally toxic and carcinogenic, where they could exemplify a major source of toxic chemicals that affect humans' health and initiate different diseases [2]. Based on the World Health Organization's (WHO) data, smoking tobacco causes around 5.4 million early deaths globally [3]. However, as the trends increase, it is expected to have 10 million deaths yearly by 2025 because of tobacco smoking [4]. These deaths are mostly related to different types of cancer (particularly lung cancer), cardiovascular diseases, and inveterate respiratory diseases. Nevertheless, the surrounding of where smoking is consumed 
is dangerous to other people, especially children, where various sicknesses could be developed just because of being close to smokers [5].

It was found that the number of individual chemicals that exist in the cigarette's smoke is unidentified but could exceed 100,000. Cigarette's pyrolysis could vary depending on the cigarette itself and/or the smoking-inhalation situation; hence the produced '"chemicals' compositions are different [6]. The natural pyridine alkaloids, such as nicotine pyridine, naturally exist in the tobacco plant's leaves. Thereby, smoking cigarettes can be replaced with smokeless habits of tobacco products, for instance, chewing the tobacco itself or its gums, in order to avoid the smoke emissions [7].

The nicotine ratio in tobacco differs from one plant to another, depending on the tobacco type and planting conditions. Here, nicotine presents around $90 \%$ of the alkaloids that exist in tobacco, which equals nearly $1.5 \mathrm{wt} . \%$ nicotine ( $8.4 \mathrm{mg}$ of nicotine for each cigarette) [8]. The other $10 \%$ of tobacco's alkaloids are nicotyrine, nornicotine, anatabine, anabasine, and myosmine [9].

The tobacco plant produces nicotine to protect itself from predators and insects since nicotine is toxic and can exterminate them. Nitrogen deficiency is a common problem in plants; therefore, nicotine is produced upon request to intensively take care of the product and keep its quality high [10]. The leaves of tobacco include more than 3800 recognized compounds, while there are 5000-6000 identified compounds during smoking. This indicates that there are nearly 2800 compounds produced from the smoking pyrolysis process that 'don't present in the plant [11].

When an individual inhales the cigarette's smoke, the nicotine condenses the dispersed particles in the lungs' fluid. This covers an area of $100 \mathrm{~m}^{2}$ surface area of the 300 million pulmonary alveoli. Nicotine normally transfers from the blood to the brain at a rapid rate of 8 10 seconds without resistance and achieves the peripheral nervous system (see figure 1). This is even faster than the intravenous injection, putting the smoker into a trance and fulfill his/her desire by adjusting the nicotine levels in the blood. However, different factors are related to nicotine uptakes, such as the inhalation volume, depth, and frequency, in addition to the smoker's body conditions [11].

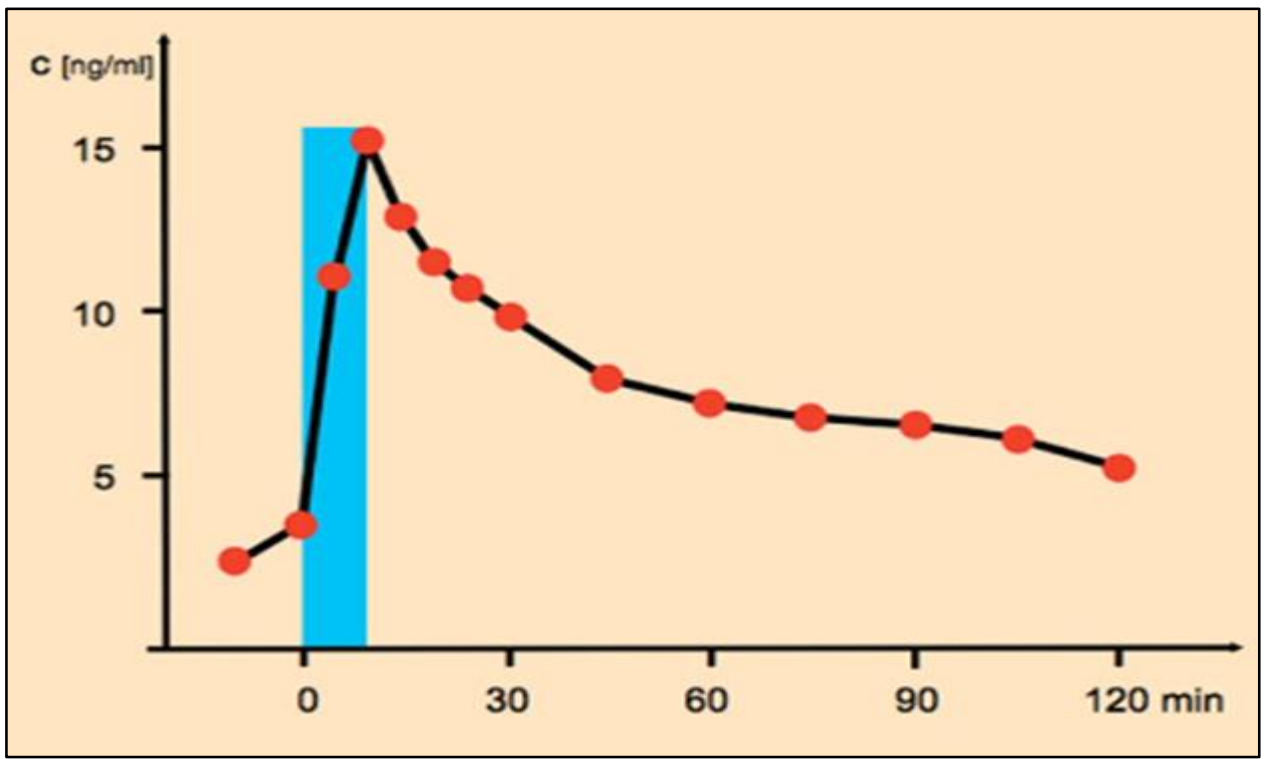

Figure 1. Nicotine concentration in blood plasma after smoking a cigarette (duration of smoking marked in blue). 
Worldwide, 15 tons/day of nicotine from tobacco is roughly consumed by smokers [12]. That alone is sufficient reason for us to consider this particular natural product more closely. This work aims to extract and determine nicotine, which is the major toxic component in tobacco leaves.

\section{Materials and Methods}

Nicotine is a hydro carbonic component that contains nitrogen (alkaloid) in its structure. It is naturally existing in many plants, including tobacco, or can be synthesized. It is an oily liquid, hygroscopic, and miscible in water. The nicotine can result in solid-water soluble salts when mixed with acids. As a free base, nicotine burns in temperatures lower than its boiling point, while $35{ }^{\circ} \mathrm{C}$ is enough for its vapors' combustion in normal conditions. However, regarding cigarette smoking, the majority of the nicotine is combusted, and enough dosage is consumed to achieve the desired condition [13].

\subsection{Sampling.}

Two samples of common brands (Iraqi cigarette and $\mathrm{Mu}$ assel) were purchased from the local market and compared to a raw tobacco sample. The raw tobacco was obtained from Sulaimaniyah/Iraq since that area is the major origin of tobacco in Iraq (see figure 2).

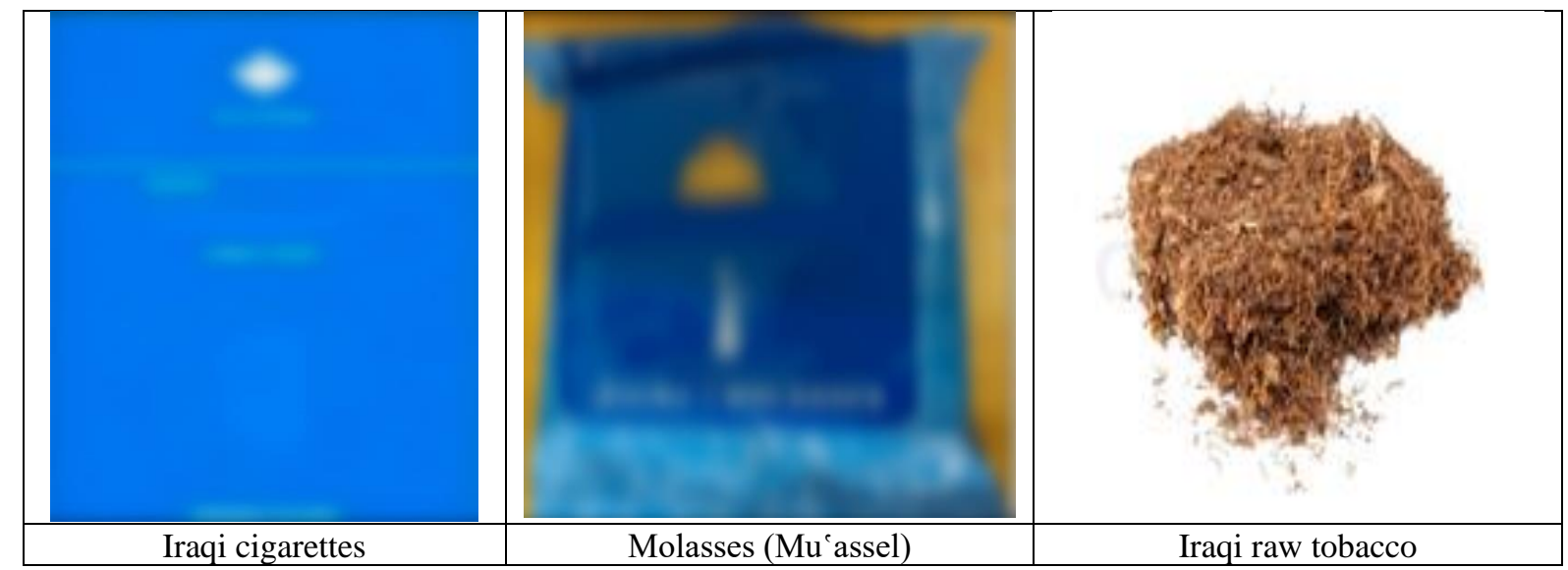

Figure 2. Samples of common brands Iraqi cigarettes, molasses, and raw tobacco. Blurred images to not promote any brand.

\subsection{Materials.}

Diethyl Ether (98\%) (Fluka), Sodium Hydroxide (Sigma Aldrich), concentrated sulfuric acid 98\% (Sigma Aldrich), Anhydrous Potassium Carbonate (BDH), Mercuric chloride (Sigma Aldrich),

\subsection{Tobacco leaves extraction.}

The extraction depends on isolating the base by dissolving the cigarettes in $\mathrm{NaOH}$, then extracting nicotine from the filtrate by ether. After the evaporation of ether, nicotine oil can be obtained. The cigarette factories remove large quantities of nicotine from tobacco leaves because of their high toxicity. Thereby, the produced oil amount is very little [14].

Five grams of all samples were weighed and placed separately into a $250 \mathrm{ml}$ beaker. About $150 \mathrm{ml}$ of $40 \% \mathrm{NaOH}$ solution was added. The contents were mixed by a magnetic stirrer thoroughly. Later, the flask with ingredients was placed on a hot plate in the fume 
chamber and warmed to about $50{ }^{\circ} \mathrm{C}$. The process was continued until a strong smell of tobacco started coming out of the flask. The Contents were cooled and filtered through Whatman 1 filter papers. The filter was washed with deionized water to ensure all the extracted solution was collected for the later step.

\subsection{Nicotine extraction.}

Nicotine is an oily yellowish-brown liquid with hygroscopic properties and easily dissolves with light petroleum, ether, and alcohol. Also, it is miscible with water in its base at a temperature range of $60-210{ }^{\circ} \mathrm{C}$ [15]. Several solvents were investigated as alkaloid extracting agents. This included isooctane, benzene, petroleum ether, and chloroform. Among the investigated hydrocarbons, Benzene showed higher satisfaction as an extracting solvent [16]. Hence, extracting the nicotine from tobacco leaves and purifying the nicotine could result in the best product. This is the reason for the extraction of nicotine by using a solvent extraction method. Later, in the extraction of nicotine as an alkaloid in tobacco, ether (could be petroleum ether) solvent is preferred to use because it is selective in dissolving the alkaloid substances. Using the right combination of ether and petroleum ether will optimize the time of extraction and nicotine production [17]. The extracted solution, obtained from section 2.3, was treated with $30 \mathrm{ml}$ of diethyl ether using a separation funnel to extract the nicotine from the alkaline solution. This was repeated twice to ensure most of the nicotine was extracted by the solvent, as shown in figure 3 (a). Then, the two filtrates were gathered in a conical flask and dried using 1 teaspoon of anhydrous potassium carbonate, as shown in figure 3 (b), and then filtered. Finally, ether was evaporated in a water bath (Avoid extra heat because nicotine is hydrolyzed by extreme heating, considering that evaporation can be done at room temperature). The extracted nicotine was a yellowish oily liquid, as illustrated in figure 3 (c).

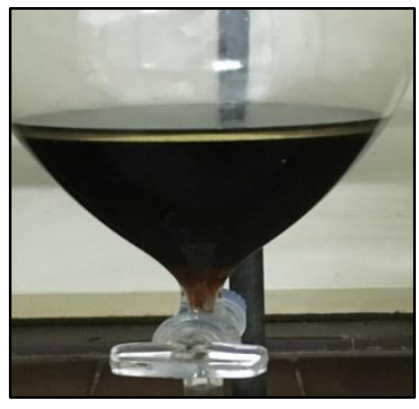

(a)

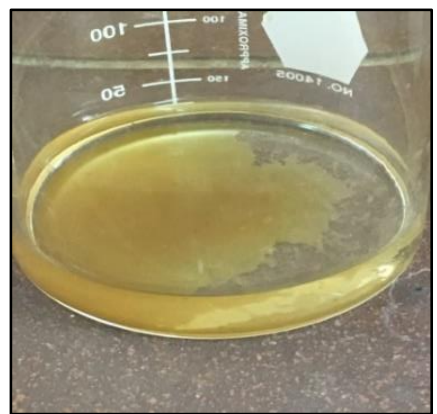

(b)

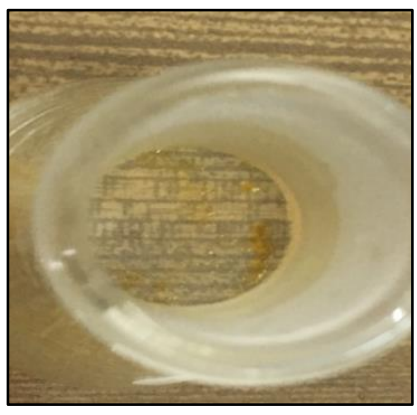

(c)

Figure 3. (a)Nicotine extraction by ether, (b) Drying by anhydrous potassium carbonate, and (c) Nicotine liquid.

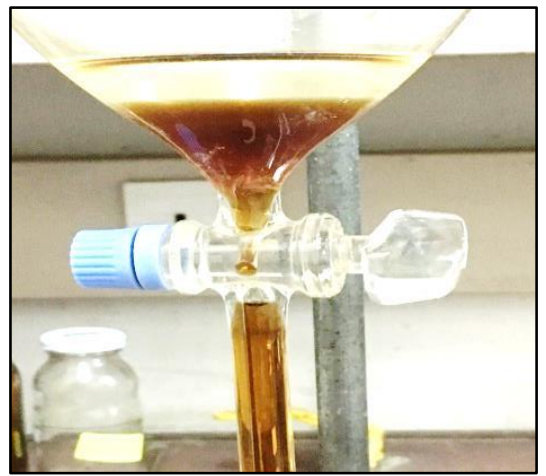

(a)

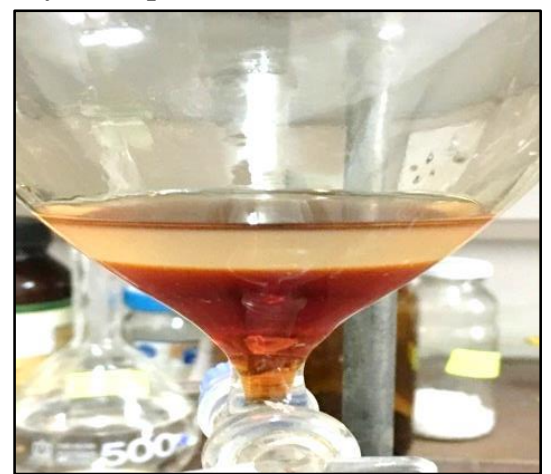

(b)

Figure 4. (a) Nicotine extraction by kerosene and (b) Nicotine extraction by sulphuric acid. 
As was mentioned earlier, nicotine is soluble in different types of solvents; this is the base for nicotine extraction from tobacco by using a solvent extraction method. The solvent choice is important because each solvent has its advantages and disadvantages [17]. As a nonexpensive and safe solvent, $30 \mathrm{ml}$ of kerosene was used to extract nicotine from the extracted solution of Iraqi tobacco obtained from sec 2.3. This process was repeated twice to ensure full nicotine extraction, as shown in figure 4 (a). The two filtrates were gathered in a separation funnel, as shown in figure 4 (b).

Nicotine was easily protonated by acid addition (figure 5) [18]. About $30 \mathrm{ml}$ of concentrated sulfuric acid was added to the collected extract in a separation funnel.
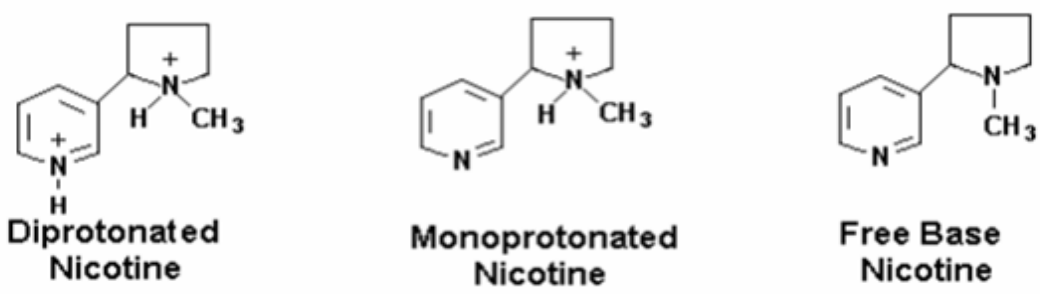

Figure 5. Mono and diprotonated nicotine.

The dissolved nicotine in sulfuric acid was placed in a ceramic crucible and heated on a hotplate until the mixture reached its boiling point. Ultimately, the crucible was covered with a Petri dish to collect nicotine as water-soluble nicotinic salt.

\section{Results and Discussion}

Although all substances in tobacco smoke affect exposed humans to some degree, nicotine is generally considered as the main responsible substance for the pharmacological responses to smoking. In many countries, tobacco smoking is recognized as a serious health hazard and a major contributing factor to deaths from several common diseases. Consequently, awareness about nicotine's toxicity is important to know tobacco-induced human diseases, in addition to reaching the essential risks accompanied by the therapeutic use of nicotine to quit smoking [19].

\subsection{Nicotine extraction with an alkaline solution.}

The chemical characteristics of nicotine are well known [8]. Pure nicotine is a colorless liquid with a characteristic acrimonious odor and boils at $246-247{ }^{\circ} \mathrm{C}$. On exposure to air and light, or even placing in the dark in a sealed bottle, the colorless or pale-yellow oily liquid nicotine turns to brownish color over time. However, the brown color nicotine is as toxic as pure colorless or pale-yellow nicotine.

Nicotine could be charged and uncharged, so the $\mathrm{pH}$ largely affects its different physicochemical processes, like toxicity, excretion, absorption, and pharmacology. Nicotine is dibasic since its pyrrolidine and pyridine nitrogens pKa values at $15^{\circ} \mathrm{C}$ are 7.84 and 3.04, respectively. It was found that when $\mathrm{pH}=7.4$ and the temperature is $37^{\circ} \mathrm{C}$, around $69 \%$ of the pyrrolidine's nitrogen ionize or gets a positive charge, while the pyridine's nitrogen is unionized [20]. Hence, the nicotine's solution $\mathrm{pH}$ significantly changes its protonated state. If the organic bases are charged, they have a hydrophilic nature; while if they don't, they are lipophilic. However, at a $\mathrm{pH}$ of 7.4, nicotine exists in two forms, so one can diffuse through the lipoprotein membranes, but the other can't. Like other alkaloids with close pKa values, Nicotine has a ratio 
of charged to uncharged forms of about 2 to 1 at a pH of 7.4. This chemical fact permits nicotine to have important biological actions.

\subsubsection{Nicotine concentration.}

Human exposure to nicotine increased in the last years due to the increasing forms of nicotine products in the market, which increased health issues. In addition to the previously available nicotine products, these products have different forms, such as smokeless tobacco and liquids for electronic nicotine delivery systems (electronic cigarettes). Unfortunately, most of these products are available worldwide without any consumption regulations. Based on previous researches and available information, a dose of 30-60 mg could kill the adult. Simply, 5 cigarettes containing $10 \mathrm{ml}$ of the diluted nicotine solution probably lead to an adult death. The $60 \mathrm{mg}$ dose could correspond to an oral median lethal dose (LD50) of $0.8 \mathrm{mg} / \mathrm{kg}$, which is smaller than the determined values for animals tested in vitro (ranging from 3.3 for mice to higher than $50 \mathrm{mg} / \mathrm{kg}$ for rats) [21]. A typical cigarette contains approximately 0.5 to $1.0 \mathrm{~g}$ of tobacco and, on average, $10 \mathrm{mg}$ of nicotine. A cigarette is typically smoked in 10 puffs and within 5 minutes. A typical smoker will absorb 1 to $2 \mathrm{mg}$ of nicotine, but absorption can range from 0.5 to $3 \mathrm{mg}[22,23]$. The elimination half-life of nicotine is 2 to 3 hours, meaning that the nicotine level in the blood decreases by one-half after a smoker stops smoking for that length of time [23].

The three samples used in this work were tested for nicotine concentration after alkaline extraction. Table 1 illustrates the calculated data for nicotine concentration.

Table 1. The calculated data for nicotine concentration.

\begin{tabular}{l|c|c|c} 
Sample & Tobacco weight $\mathbf{( g )}$ & Nicotine weight $\mathbf{( g )}$ & Nicotine $(\boldsymbol{\%})$ \\
\hline Iraqi cigarette & 5.00 & 0.08 & 1.6 \\
\hline Molasses & 5.00 & 0.05 & 1.0 \\
\hline Iraqi raw tobacco & 5.00 & 0.12 & 2.4
\end{tabular}

It is clear from table 1 that Iraqi tobacco contains more nicotine than Iraqi cigarettes. Both are higher than shisha tobacco (molasses) since it contains large amounts of flavors and other non-tobacco ingredients.

\subsubsection{FT-IR analysis for nicotine.}

FT-IR (ALPHA-Bruker) was used for instrumental analysis, the FT-IR spectrum of nicotine was illustrated in figure 6 [24].

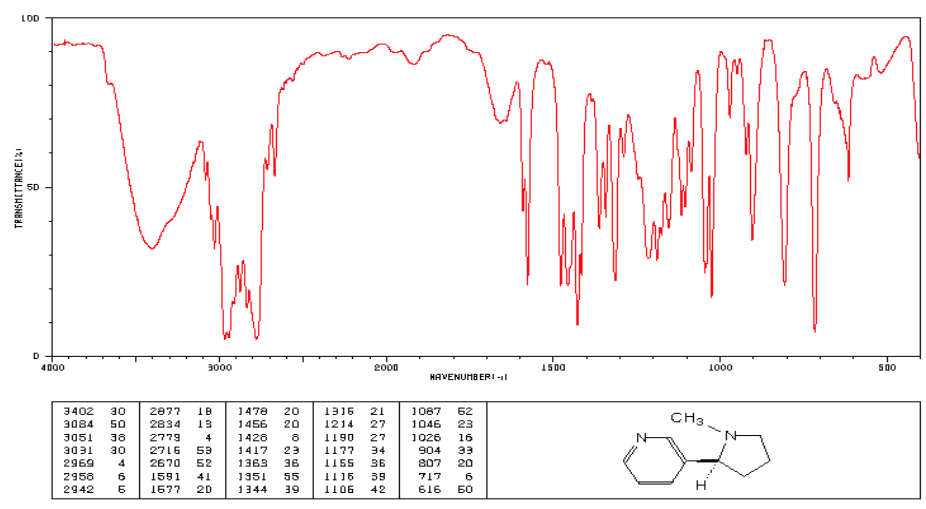

Figure 6. FT-IR spectrum for nicotine. 
From the figure, several peaks can be noticed, which characterize the different chemical functions of nicotine:

- Around $3400 \mathrm{~cm}^{-1}$, a large water peak can be seen (it deals with a liquid film).

- Between 2970 and $2780 \mathrm{~cm}^{-1}$ : C-H stretching.

- The peak at $1677 \mathrm{~cm}^{-1}$ : aromatic $\mathrm{C}=\mathrm{N}$ double bond stretching.

- The peak at $1691 \mathrm{~cm}^{-1}$ : aromatic $\mathrm{C}=\mathrm{C}$ double bond stretching.

- The peaks at $717 \mathrm{~cm}^{-1}$ and $904 \mathrm{~cm}^{-1}$ correspond to the out-of-plane bending of the $\mathrm{C}-\mathrm{H}$ bond of the mono-substituted pyridinic cycle.

FT-IR analysis was carried out for the extracted nicotine for one tobacco sample (Sumer Iraqi cigarette). Figures 7 shows the obtained FT-IR spectrum for the extracted nicotine from Sumer cigarette (the Iraqi cigarette) tobacco.

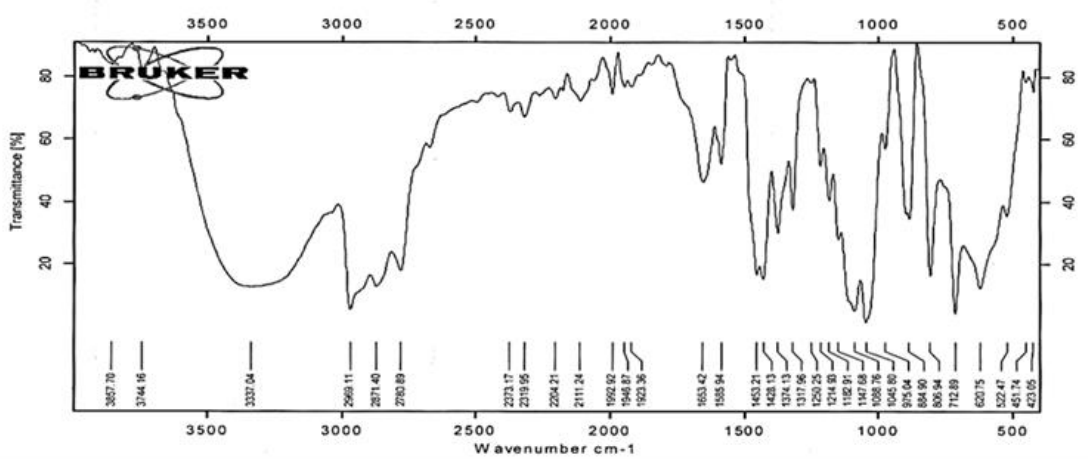

Figure 7. FT-IR spectrum for the extracted nicotine from Iraqi cigarette tobacco.

From figure 7, several peaks can be observed other than the original sample, which characterizes the different chemical functions of nicotine:

- Around $3337 \mathrm{~cm}^{-1}$, we can see the large peak of water.

- Between 2969 and $2780 \mathrm{~cm}^{-1}$ : C-H stretching.

- The peak at $1585 \mathrm{~cm}^{-1}$ : aromatic $\mathrm{C}=\mathrm{N}$ double bond stretching.

- The peak at $1653 \mathrm{~cm}^{-1}$ : aromatic $\mathrm{C}=\mathrm{C}$ double bond stretching.

- The peaks at $712 \mathrm{~cm}^{-1}$ and $884 \mathrm{~cm}^{-1}$ correspond to the out-of-plane bending of the $\mathrm{C}-\mathrm{H}$ bond of the monosubstituted pyridinic cycle.

To ensure that most of the nicotine in tobacco for the tested sample was extracted, the alkaline solution was tested after nicotine extraction by diethyl ether (figure 8 shows FT-IR analysis for the alkaline solution after nicotine extraction). FT-IR also tested the solid precipitate from filtration tobacco from alkaline solution to ensure that all nicotine was extracted (figure 9 shows the obtained spectrum for tobacco precipitate after nicotine extraction).

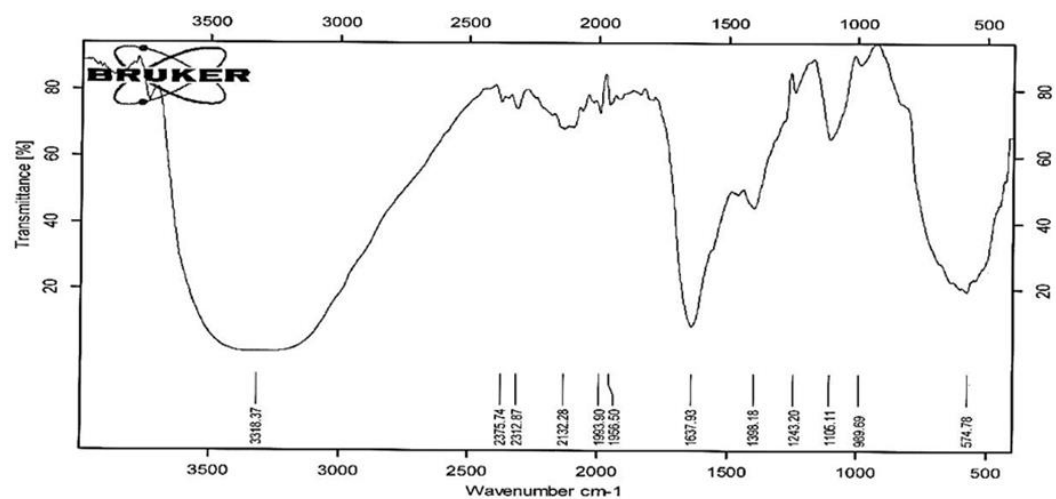

Figure 8. FT-IR analysis for the alkaline solution after nicotine extraction. 


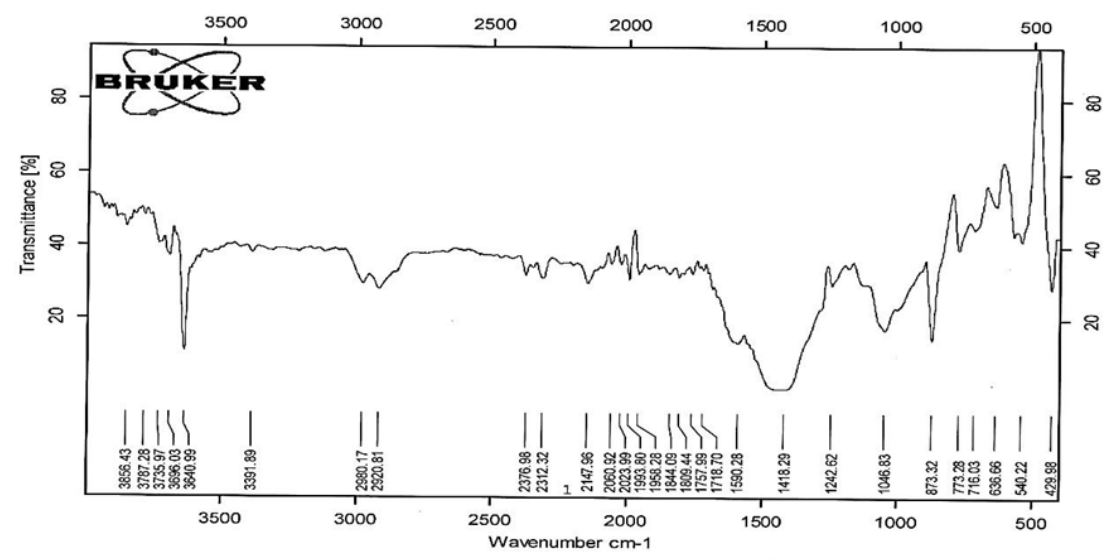

Figure 9. FT-IR spectrum for tobacco precipitate after nicotine extraction.

It is clear from figures 8 and 9 that the tested samples were freed from nicotine, and most of nicotine was extracted.

\subsubsection{Ultraviolet analysis for nicotine.}

The spectrophotometric analysis was conducted for nicotine in this research. However, nicotine in tobacco was preliminarily analyzed at the ATF National Laboratory Center using spectrophotometry. This test has many advantages, including rapid analysis, ease of use, capability, well establishment, and reliability. On the other hand, there are some disadvantages, where the main one is related to tobacco's alkaloids structure. This means that the compounds' UV absorption take place in the $260 \mathrm{~nm}$ region, which interferes with the absorbance of nicotine. Nevertheless, the ATF regulated tobacco products and considered all alkaloids as nicotine. It is important to ensure that the purity of nicotine is high to obtain the ideal UV absorbance measurements and provide valuable information about the standard nicotine chemistry. Nicotine is strongly absorbed in the UV at about $260 \mathrm{~nm}$, as shown in figure 10, this absorbance depends on the $\mathrm{pH}$ of the used solvent. Figure 11 shows the UV spectra of nicotine in sodium phosphate buffer, $\mathrm{pH} 6.9$ (A), deionized water (B), and sodium phosphate buffer, $\mathrm{pH} 2.5$ [25].

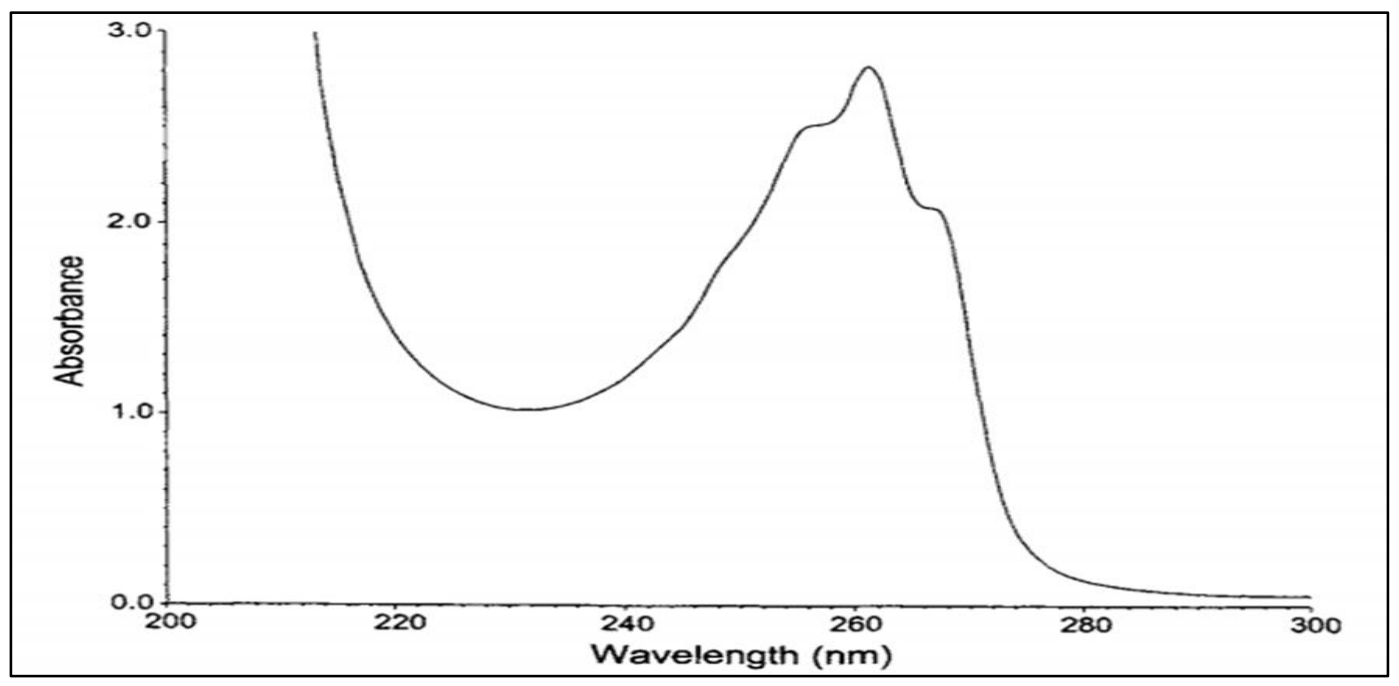

Figure 10. UV absorbance spectrum of nicotine. 


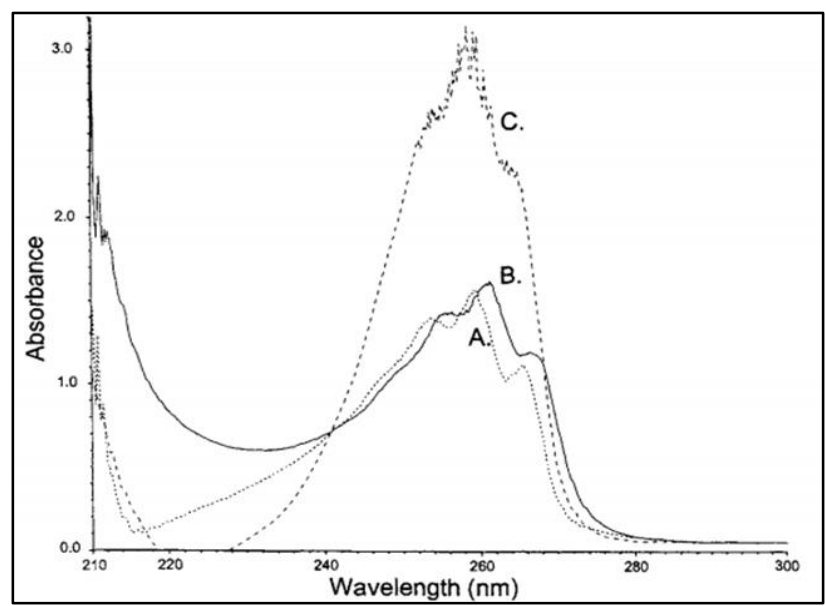

Figure 11. UV absorbance spectra of nicotine: (A) in $10 \mathrm{mM}$ sodium phosphate buffer, pH 6.9, (B) in distilled, deionized water, and $(\mathrm{C})$ in $25 \mathrm{mM}$ sodium phosphate buffer, $\mathrm{pH} 2.5$.

UV analysis was carried out for the Iraqi cigarette tobacco sample's extracted nicotine using an Ultraviolet spectrometer (Shimadzu UV-1650PC). Figures 12 shows the obtained UV spectrum of the nicotine dissolved in diethyl ether at $\mathrm{pH}=5.5$.

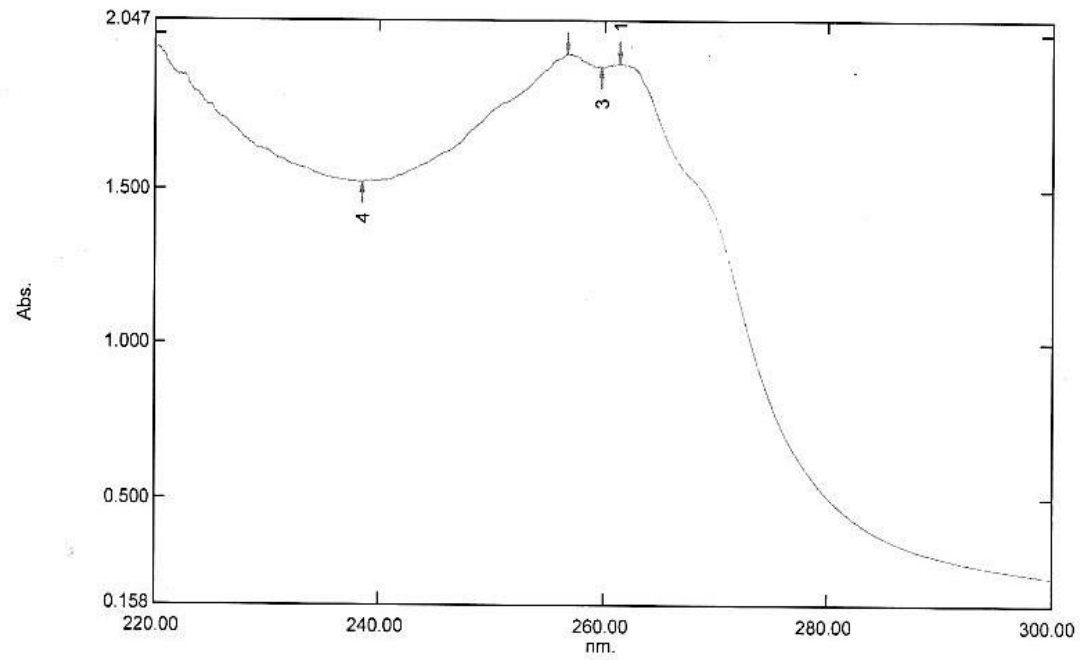

Figure 12. UV absorbance spectrum of nicotine for Iraqi cigarette tobacco.

UV absorbance for tobacco nicotine is absorbed in the $260 \mathrm{~nm}$ region. Alkaloids, such as nornicotine, are also absorbed in the $260 \mathrm{~nm}$ region, thus interfering with the absorbance of nicotine. Figure 12 shows the absorbance at $259.80 \mathrm{~nm}$ for the extracted nicotine in the presence of other alkaloids in the sample.

3.1.4. Microscopic examination for nicotine.

The heterocyclic nitrogen compounds are considered among the notable bio-ligands modules in bioinorganic and structural chemistry [26]. The widely studied type of these compounds is the six-membered heterocyclic, such as nitrogen pyridine, 2, 2'-bipyridine, and $2,2^{\prime}: 6$ ', 2"-terpyridine. The five-membered ligands heterocyclic nitrogen compounds, such as pyrazoles and imidazoles, were also intensively utilized in inorganic chemistry $[26,27]$. Only a few studies about the nicotine's organic and inorganic chemistry and its derivatives were reported by exploring the literature. At the same time, the majority report the preparation of new substituted nicotine derivatives and analogs due to their pharmacological applications as 
potential chiral coordination ligands. Three crystal structures of metal complexes of nicotine had been previously reported. In these studies, the first crystal structure of a mercuric complex of nicotine was determined, describing a structure in which nicotine acts as a bridging ligand between the tetrahedral $\mathrm{Hg}$ (II) centers, forming a one-dimensional coordination polymer. The quantum-mechanical estimations aid in elucidating the experimental results as they give data on the interactions energy modes of $\mathrm{Cu}$ (II) with nicotine [28, 29].

Nicotine can be examined as a crystal shape after adding mercuric chloride to the nicotine extract with methanol, a flowery-shaped crystal formed [25]. Diethyl ether solution with the extracted nicotine from Sumer tobacco was examined to shape crystals after adding mercuric chloride. Figure 13 shows the obtained crystals shape examined under the microscope for the extracted nicotine. Nicotine acts as a bridging ligand between tetrahedral $\mathrm{Hg}$ (II) centers, forming a one-dimensional coordination polymer [30].
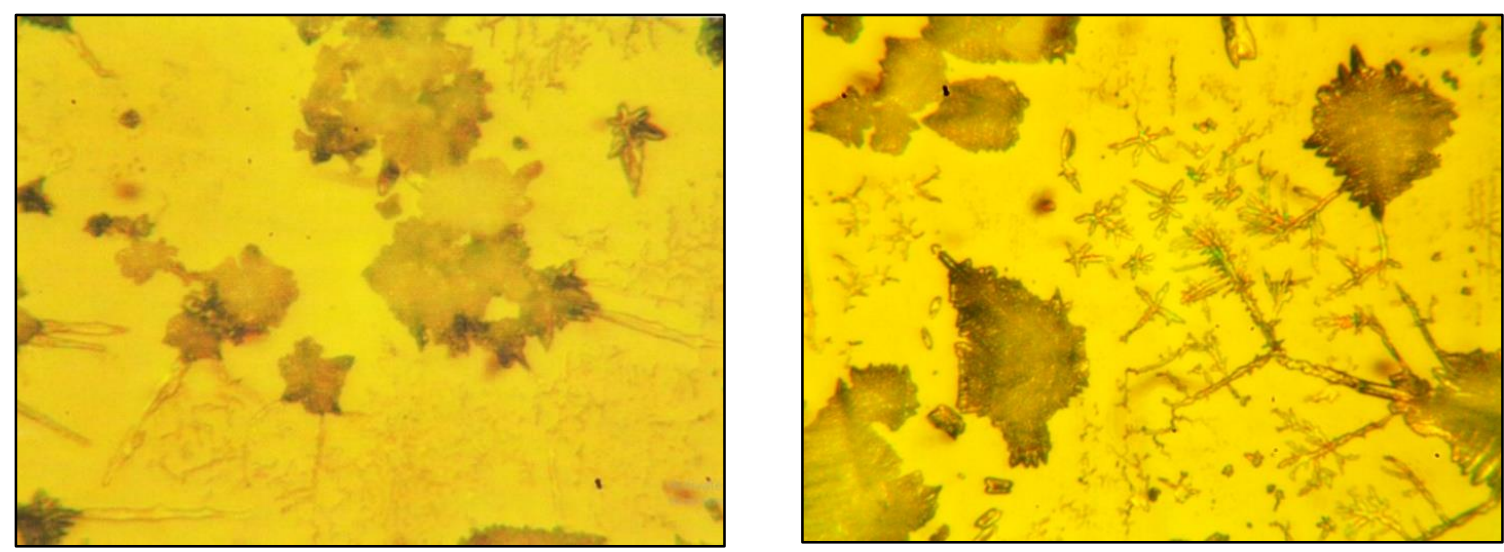

Figure 13. Flowery crystal shape of nicotine bridging ligand with mercuric chloride.

3.1.5. Nicotine extraction with kerosene.

Nicotine is a Diprotic Base with '" 'pKa's of 3.12 (pyridine ring) and 8.02 (pyrrolidine ring) (see figure 14).

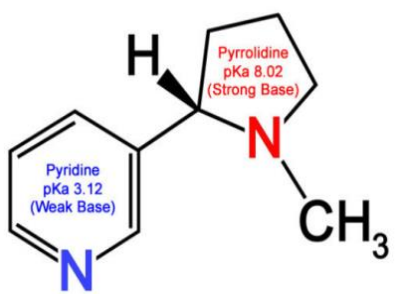

Figure 14. Nicotine as a diprotic base.

The nicotine molecule contains two nitrogen atoms capable of accepting a proton. The pyrrolidine nitrogen is considerably more basic than the pyridine nitrogen [31]. As a cheap and safe solvent, kerosene was used to extract nicotine from the extracted solution for Iraqi tobacco obtained from sec 2.3. Nicotine solution with kerosene dissolved in sulfuric acid, nicotine as water-soluble, nicotinic salt was obtained as dark brown drops.

\subsection{Nicotine extraction with deionized water.}

Nicotine is soluble in water and non-polar solvents. The same method of nicotine extraction was carried out using deionized water instead of the alkaline solution. Figure 15 shows the FT-IR spectrum for nicotine, clearly showing the absence of the major nicotine peaks 
in the obtained spectrum due to the slight nicotine solubility in neutral water compared with alkaline. Also, the wide peak of water around $3251 \mathrm{~cm}^{-1}$ may affect the other spectrum peaks.

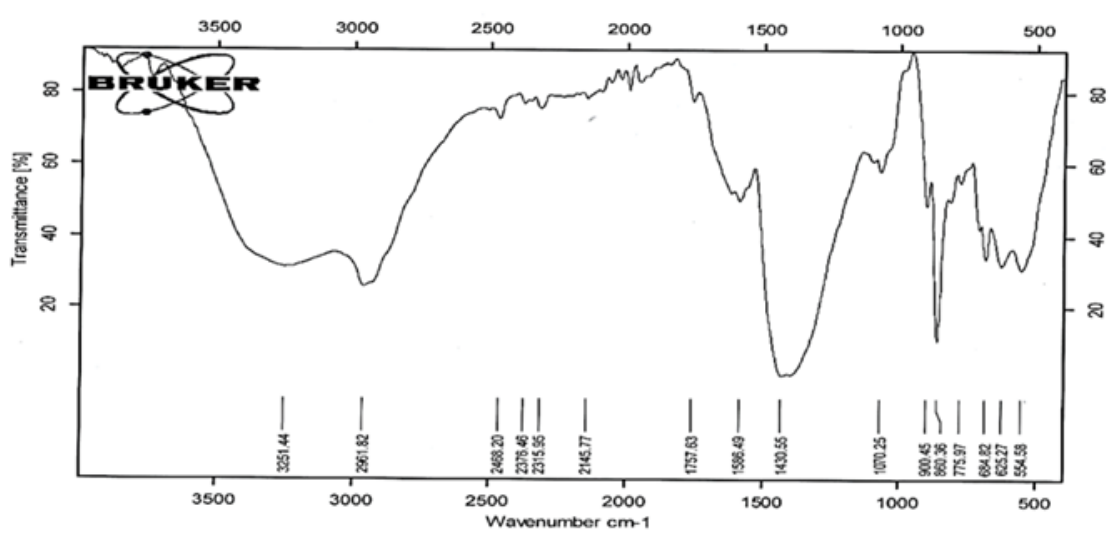

Figure 15. FT-IR spectrum for the extracted nicotine by water from Iraqi cigarette tobacco.

\section{Conclusions}

Because of its toxicity and harmful effects on human lives, nicotine is the most important compound to be determined in tobacco. This work aimed to extract and determine nicotine, which is the major toxic component in tobacco leaves, using different methods. Tobacco cigarettes, tobacco shisha (called molasses), and raw tobacco were investigated and compared. All of the extracted methods were based on water extraction as a polar solvent and non-polar solvents. Water, as a neutral polar solvent, showed a limited extraction activity of nicotine from tobacco leaves. Because nicotine is a dibasic compound, an alkaline solution $(40 \% \mathrm{NaOH})$ was used to extract nicotine from tobacco leaves for the selected samples. Further solvents extraction by non-polar solvents was carried out to extract nicotine from the alkaline solution and prepared for spectrometric analysis. FT-IR and UV analysis showed that the oily substance extracted from tobacco leaves is nicotine. Results were confirmed by the microscopic examination for the extracted nicotine after adding mercuric chloride to form flowery shape complex crystals of nicotine bridging ligand with mercuric chloride. Promising data was obtained when using ground tobacco leaves for water extraction, which may be recommended as a future work for nicotine extraction.

\section{Funding}

This research received no external funding.

\section{Acknowledgments}

The authors like to thank their affiliations for partially supporting this work

\section{Conflicts of Interest}

The authors declare no conflict of interest.

\section{References}

1. Percoco, G.; Patatian, A.; Eudier, F.; Grisel, M.; Bader, T.; Lati, E.; Savary, G.; Picard, C.; Benech, P. Impact of cigarette smoke on physical-chemical and molecular proprieties of human skin in an ex vivo model. Experimental Dermatology 2020, https://doi.org/10.1111/exd.14192. 
2. Elmassry, M.M.; Zayed, A.; Farag, M.A. Gut homeostasis and microbiota under attack: impact of the different types of food contaminants on gut health. Critical Reviews in Food Science and Nutrition 2020, 126, https://doi.org/10.1080/10408398.2020.1828263.

3. WHO Report on the Global Tobacco Epidemic. the MPOWER Package; WHO: Geneva, Switzerland, 2008.

4. Hatsukami, D.K.; Stead, L.F.; Gupta, P.C. Tobacco addiction. The Lancet 2008, 371, 2027-2038, https://doi.org/10.1016/S0140-6736(08)60871-5.

5. Harmeet Singh, R.; Jahnavi, M.; Amrit Pal Singh, H. Vaping Versus Smoking: A Quest for Efficacy and Safety of E-cigarette. Current Drug Safety 2018, 13, 92-101, https://doi.org/10.2174/1574886313666180227110556.

6. Wakeham, H.; Schmeltz, I. Symposium on the composition of tobacco and tobacco smoke. American Chemical Society Meeting, Washington DC. Plenum Press, New York, 1972; pp. 1-20.

7. Kaur, G.; Muthumalage, T.; Rahman, I. Mechanisms of toxicity and biomarkers of flavoring and flavor enhancing chemicals in emerging tobacco and non-tobacco products. Toxicology Letters 2018, 288, 143155, https://doi.org/10.1016/j.toxlet.2018.02.025.

8. Padhiary, S.; Samal, D.; Khandayataray, P.; Murthy, M.K. A systematic review report on tobacco products and its health issues in India. Reviews on environmental health 2020, https://doi.org/10.1515/reveh-20200037.

9. Liu, X.; Joza, P.; Rickert, B. Analysis of Nicotine and Nicotine-Related Compounds in Electronic Cigarette Liquids and Aerosols by Liquid Chromatography-Tandem Mass Spectrometry. Beiträge zur Tabakforschung International/Contributions to Tobacco Research 2017, 27, 154-167, https://doi.org/10.1515/cttr-20170016.

10. Sabine, S.; Klaus, R. The Chemistry of Tobacco - Part 3. Wiley-VCH Verlag GmbH \& Co., 2014; https://doi.org/10.1002/chemv.201400117.

11. Sabine, S.; Klaus, R. The Chemistry of Tobacco - Part 4. Wiley-VCH Verlag GmbH \& Co., 2015; https://doi.org/10.1002/chemv.201400135.

12. Sabine, S.; Klaus, R. The Chemistry of Tobacco - Part 2. Wiley-VCH Verlag GmbH \& Co., 2014; https://doi.org/10.1002/chemv.201400107.

13. Kurek, J. Introductory Chapter: Alkaloids - Their Importance in Nature and for Human Life. IntechOpen. 2019; http://dx.doi.org/10.5772/intechopen.85400.

14. Qayyum, I.; Fazal-Ur-Rehman, M.; Shamsuddin, I. Extraction of Nicotine (3-(1-methyl-2-pyrrolidinyl) pyridine) from Tobacco Leaves Separated from Gold Live Classic BrandTM Cigarettes by Solvent Extraction Approach and Characterization Via IR Analysis. Biosciences Biotechnology Research Asia 2018, 15, http://dx.doi.org/10.13005/bbra/2688.

15. Luttrell, W.E.; Vogel, H.F. Nicotine. Journal of Chemical Health \& Safety 2014, 21, 39-41, https://doi.org/10.1021/acs.chas.8b21408.

16. Truong, D.; Nguyen, D.; Thuy Anh Ta, N.; Vo Bui, A.; Ha Do, T.; Nguyen, H. Evaluation of the Use of Different Solvents for Phytochemical Constituents, Antioxidants, and In Vitro Anti-Inflammatory Activities of Severinia buxifolia. Journal of Food Quality 2019, 2019, https://doi.org/10.1155/2019/8178294.

17. Arie, F.; Susinggih, W.; Arif, S. Optimization of Nicotine Extraction in Tobacco Leaf (Nicotiana tabacumL.) :(Study: Comparison of Ether and Petroleum Ether). The International Conference on Chemical Engineering UNPAR, 2013.

18. Kuhn, T.; Krudysz, M.; Zhu, Y.; Fine, P.M.; Hinds, W.C.; Froines, J.; Sioutas, C. Volatility of indoor and outdoor ultrafine particulate matter near a freeway. Journal of Aerosol Science 2005, 36, 291-302, https://doi.org/10.1016/j.jaerosci.2004.09.006.

19. Giulietti, F.; Filipponi, A.; Rosettani, G.; Giordano, P.; Iacoacci, C.; Spannella, F.; Sarzani, R. Pharmacological Approach to Smoking Cessation: An Updated Review for Daily Clinical Practice. High Blood Pressure \& Cardiovascular Prevention 2020, 27, 349-362, https://doi.org/10.1007/s40292-02000396-9.

20. Domino, F. Behavioral, electrophysiological, endocrine, and skeletal muscle actions of nicotine and tobacco smoking. In: Electrophysiological effects of nicotine. Remond, A.; Izard, C. (Eds.), Amsterdam: Elsevier/North Holland Biomedical Press, 1979; pp.133-146.

21. Mayer, B. How much nicotine kills a human? Tracing back the generally accepted lethal dose to dubious self-experiments in the nineteenth century. Archives of Toxicology 2014, 88, 5-7, https://doi.org/10.1007/s00204-013-1127-0.

22. Karan, D.; Dani, A.; Benowitz, L. The pharmacology of nicotinedependence. In: Principles of addiction medicine. 3rd ed. Washington,DC, American Society of Addiction Medicine, 2003; pp. 225-248.

23. Jacob, P., III; St. Helen, G.; Yu, L.; Nardone, N.; Havel, C.; Cheung, P.; Benowitz, N.L. Biomarkers of Exposure for Dual Use of Electronic Cigarettes and Combustible Cigarettes: Nicotelline, NNAL, and Total Nicotine Equivalents. Nicotine \& Tobacco Research 2020, 22, 1107-1113, https://doi.org/10.1093/ntr/ntz235.

24. Michelina, C.; Naviglio, D.; Gallo, M.; Severina, P. FT-IR and GC-MS analyses of an antioxidant leaf essential oil from sage plants cultivated as an alternative to tobacco production. Journal of Essential Oil Research 2019, 31, 138-144, https://doi.org/10.1080/10412905.2018.1540364. 
25. Gorrod, W. Analytical Determination of Nicotine and Related Compounds and Their Metabolites. Elsevier. 1999.

26. Natori, Y.; Imahori, T.; Yoshimura, Y. Development of stereoselective synthesis of biologically active nitrogen-heterocyclic compounds: Applications for syntheses of natural product and organocatalyst. J Synth Org Chem 2016, 74, 335-349, https://doi.org/10.5059/YUKIGOSEIKYOKAISHI.74.335.

27. Khan, M.F.; Alam, M.M.; Verma, G.; Akhtar, W.; Akhter, M.; Shaquiquzzaman, M. The therapeutic voyage of pyrazole and its analogs: A review. European Journal of Medicinal Chemistry 2016, 120, 170-201, https://doi.org/10.1016/j.ejmech.2016.04.077.

28. Flammia, D.; Dukat, M.; Damaj, M.I.; Martin, B.; Glennon, R.A. Lobeline: structure-affinity investigation of nicotinic acetylcholinergic receptor binding. $J$ Med Chem 1999, 42, 3726-3731, https://doi.org/10.1021/jm990286m.

29. Uçar, I.; Karabulut, B.; Bulut, A.; Büyükgüngör, O. Crystal structure and EPR studies of mixed ligand complex of cobalt(II) with saccharin and ethylisonicotine. Spectrochim Acta A Mol Biomol Spectrosc 2008, 71, 1239-1245, https://doi.org/10.1016/j.saa.2008.03.035.

30. Ahmed, F. Metal Complexes of Nicotine: A Group of Negligible Compounds. Pelagia Research Library, Der Chemica Sinica 2017, 8, 413-420.

31. Ciolino, L.A.; Turner, J.A.; McCauley, H.A.; Smallwood, A.W.; Yi, T.Y. Optimization study for the reversed-phase ion-pair liquid chromatographic determination of nicotine in commercial tobacco products. Journal of Chromatography A 1999, 852, 451-463, https://doi.org/10.1016/S0021-9673(99)00639-1. 